\title{
PROGRESS ON MODEL ATMOSPHERES AND LINE DATA
}

\author{
ROBERT L. KURUCZ \\ Harvard-Smithsonian Center for Astrophysics, 60 Garden St., \\ Cambridge, MA 02138, USA
}

\begin{abstract}
I discuss errors in theory and in interpreting observations that are produced by the failure to consider resolution in space, time, and energy. I discuss convection in stellar model atmospheres and in stars. One dimensional convective models can never work well, but the errors in predicted diagnostics for temperature, gravity, and abundances can be calibrated. I discuss the variation of microturbulent velocity with depth, effective temperature, and gravity. These variations must be dealt with in computing models and grids and in any type of photometric calibration. I describe the state of the art in computing a model atmosphere and complete spectrum for stars with arbitrary abundances. I have made no significant progress on atomic or molecular line data for the last two years, but I will soon begin large scale production. I will continue to distribute the results on CD-ROMs, and I will make them available on the World Wide Web.
\end{abstract}

\section{Introduction}

For the past two years I have been preoccupied with convection because the model atmospheres are now good enough to show shortcomings in the convective treatment. Here I will outline what I have learned. I will mainly list the conclusions I have come to from examining individual convective models and from examining grids of convective models as a whole. Eighteen figures illustrating the points made here can be found in Kurucz (1996a).

Every observation, measurement, model, and theory has seven characteristic numbers: resolution in space, in time, and in energy, and minimum and maximum energy. Many people never think about these resolutions. A low resolution physics cannot be used to study something in which the physical process of interest occurs at high resolution unless the high resolution effects average out when integrated over the resolution bandpasses.

T.R. Bedding et al. (eds.),

Fundamental Stellar Properties: The Interaction between Observation and Theory, 217-226.

(C) 1997 IAU. Printed in the Netherlands. 
What does the sun, or any convective atmosphere, actually look like? We do not really know yet. There is a very simplified three-dimensional radiation-hydrodynamics calculation discussed in the review by Chan, Nordlund, Steffen, and Stein (1991). It is consistent with the high spatial and temporal resolution observations shown in the review by Topka and Title (1991). Qualitatively, there is cellular convection with relatively slowly ascending, hot, broad, diverging flows that turn over and merge with their neighbors to form cold, rapidly descending, filamentary flows that diffuse at the bottom. The filling factor for the cold downward flowing elements is small. The structure changes with time. Nordlund and Dravins (1990) discuss four similar stellar models with many figures. Every one-dimensional mixing-length convective model is based on the assumption that the convective structure averages away so that the emergent radiation depends only a one-dimensional temperature distribution.

There is a solar flux atlas (Kurucz, Furenlid, Brault, and Testerman 1984) that Ingemar Furenlid caused to be produced because he wanted to work with the sun as a star for comparison to other stars. The atlas is pieced together from eight Fourier transform spectrograph scans, each of which was integrated for two hours, so the time resolution is two hours for a given scan. The $\mathrm{x}$ and $\mathrm{y}$ resolutions are the diameter of the sun. The $\mathrm{z}$ resolution (from the formation depths of features in the spectrum) is difficult to estimate. It depends on the signal-to-noise and the number of resolution elements. The first is greater than 3000 and the second is more than one million. It may be possible to find enough weak lines in the wings and shoulders of strong lines to map out relative positions to a few kilometers. Today I think it is to a few tens of kilometers. The resolving power is on the order of 522,000. This is not really good enough for observations made through the atmosphere because it does not resolve the terrestrial lines that must be removed from the spectrum. (In the infrared there are many wavelength regions where the terrestrial absorption is too strong to remove.) The sun itself degrades its own flux spectrum by differential rotation and macroturbulent motions. The energy range of the atlas is from 300 to $1300 \mathrm{~nm}$, essentially the range where the sun radiates most of its energy.

This solar atlas is of higher quality than any stellar spectrum taken thus far but still needs considerable improvement. If we have difficulty interpreting these data, it can only be worse for other stars where the spectra are of lower quality by orders of magnitude.

To analyze this spectrum, or any other spectrum, we need a theory that works at a similar resolution or better. We use a plane parallel, onedimensional theoretical or empirical model atmosphere that extends in $\mathrm{z}$ through the region where the lines and continuum are formed. The onedimensional model atmosphere represents the space average of the convec- 
tive structure over the whole stellar disk (taking account of the center-tolimb variation) and the time average over hours. It is usually possible to compute a model that matches the observed energy distribution around the flux maximum. However, to obtain the match it is necessary to adjust a number of free parameters: effective temperature, surface gravity, microturbulent velocity, and the mixing-length-to-scale-height-ratio in the one-dimensional convective treatment. The microturbulent velocity parameter also produces an adjustment to the line opacity to make up for missing lines. Since much of the spectrum is produced near the flux maximum, at depths in the atmosphere where the overall flux is produced, averaging should give good results. The parameters of the fitted model may not be those of the star, but the radiation field should be like that of the star. The sun is the only star where the effective temperature and gravity are accurately known. In computing the detailed spectrum, it is possible to adjust the line parameters to match all but the centers of the strongest lines. Since very few lines have atomic data known accurately enough to constrain the model, a match does not necessarily mean that the model is correct.

\section{Convective bullets}

From plots of the convective flux and velocity for grids of models I have identified three types of convection in stellar atmospheres:

- normal strong convection where the convection is continuous from the atmosphere down into the underlying envelope. Convection carries more than $90 \%$ of the flux. Stars with effective temperatures $6000 \mathrm{~K}$ and cooler are convective in this way as are stars on the main sequence up to $8000 \mathrm{~K}$. At higher temperature the convection carries less of the total flux and eventually disappears starting with the lowest gravity models. Intermediate gravities have intermediate behavior. Abundances have to be uniform through the atmosphere into the envelope. The highly convective models seem to be reasonable representations of real stars, except for the shortcomings cited below.

- atmospheric layer convection where. as convection weakens, the convection zone withdraws completely up from the envelope into the atmosphere. There is zero convection at the bottom of the atmosphere. Abundances in the atmosphere are decoupled from abundances in the envelope. For mixinglength models the convection zone is limited at the top by the Schwarzschild criterion to the vicinity of optical depth 1 or 2 . The convection zone is squashed into a thin layer. In a grid, this layer continues to carry significant convective flux for about $500 \mathrm{~K}$ in effective temperature beyond the strongly convective models. There is no common-sense way in which to have convective motions in a thin layer in an atmosphere. The solution is that 
the Schwarzschild criterion does not apply to convective atmospheres. The derivatives are defined only in one dimensional models. A real convective element has to decide what to do on the basis of local three-dimensional derivatives, not on means. These thin-layer-convective model atmospheres may not be very realistic.

- plume convection. Once the convective flux drops to the percent range, cellular convection is no longer viable. Either the star becomes completely radiative, or it becomes radiative with convective plumes that cover only a small fraction of the surface in space and time. Warm convective material rises and radiates. The star has rubeola. The plumes dissipate and the whole atmosphere relaxes downward. There are no downward flows. The convective model atmospheres are not very realistic except when the convection is so small as to have negligible effect, i.e. the model is radiative. The best approach may be simply to define a star with less than, say, $1 \%$ convection as radiative. The error will probably be less than using mixing-length model atmospheres.

Using a one-dimensional model atmosphere to represent a real convective atmosphere for any property that does not average in space and time to the one-dimensional model predictions produces systematic errors. The Planck function, the Boltzman factor, and the Saha equation are functions that do not average between hot and cold convective elements. We can automatically conclude that one-dimensional convective models must predict the wrong value for any parameter that has strong exponential temperature dependence from these functions.

Starting with the Planck function, the ultraviolet photospheric flux in any convective star must be higher than predicted by a one-dimensional model (Bikmaev 1994). Then, by flux conservation, the flux redward of the flux maximum must be lower. It is fit by a model with lower effective temperature than that of the star.

The following qualitative predictions result from the exponential falloff of the flux blueward of the flux maximum:

- the Balmer continuum in all convective stars is higher than predicted by a one-dimensional model;

- in G stars, including the sun, the discrepancy reaches up to about $400 \mathrm{~nm}$; - in K stars it reaches to about $500 \mathrm{~nm}$. This flux "distortion" may be responsible for Short and Lester's (1994) problems with the ultraviolet flux of Arcturus;

- in M stars the whole Paschen continuum is higher;

- all ultraviolet photoionization rates at photospheric depths are higher in real stars than computed from one-dimensional models;

- flux from a temperature minimum and a chromospheric temperature rise masks the increased photospheric flux in the ultraviolet; 
- the spectrum predicted from a one-dimensional model for the exponential falloff region, and abundances derived therefrom, are systematically in error;

- limb-darkening predicted from a one-dimensional model for the exponential falloff region is systematically in error;

- convective stars produce slightly less infrared flux than do one-dimensional models.

The Boltzman factor is extremely temperature sensitive for highly excited levels:

- the strong Boltzmann temperature dependence of the second level of hydrogen implies that the Balmer line wings are preferentially formed in the hotter convective elements. A one-dimensional model that matches Balmer line wings has a higher effective temperature than the real star;

- the same is true for all infrared hydrogen lines.

The Saha equation is safe only for the dominant species:

- neutral atoms for an element that is mostly ionized are the most dangerous because (in LTE) they are much more abundant in the cool convective elements. When $\mathrm{Fe}$ is mostly ionized the metallicity determination from $\mathrm{Fe}$ I can be systematically offset and can result in a systematic error in the assumed evolutionary track and age.

- in the sun convection may account for the remaining uncertainties with Fe I found by Blackwell, Lynas-Gray, and Smith (1995);

- the most striking case is the large systematic error in Li abundance determination in extreme Population II G subdwarfs. The abundance is determined from the Li I D lines which are formed at depths in the highly convective atmosphere where $\mathrm{Li}$ is $99.94 \%$ ionized (Kurucz 1995b);

- K giant abundances also have systematic errors that arise from similar mechanisms.

- molecules with high dissociation energies such as $\mathrm{CO}$ are also much more abundant in the cool convective elements. The $\mathrm{CO}$ fundamental line cores in the solar infrared are deeper than any one-dimensional model predicts (Ayres and Testerman 1981) because the cooler convective elements that exist only a short time have more $\mathrm{CO}$ than the mean model.

Given all of these difficulties, how should we proceed? One-dimensional model atmospheres can never reproduce real convective atmospheres. The only practical procedure is to compute grids of model atmospheres, then to compute diagnostics for temperature, gravity, abundances, etc., and then to make tables of corrections. Say, for example, in using the $\mathrm{H} \alpha$ wings as a diagnostic of effective temperature in $\mathrm{G}$ stars, the models may predict effective temperatures that are $100 \mathrm{~K}$ too high. So if one uses an $\mathrm{H} \alpha$ temperature scale it has to be corrected by $100 \mathrm{~K}$ to give the true answer. Every temperature scale by any method has to be corrected in some way. 
Unfortunately, not only is this tedious, but it is very difficult or impossible because no standards exist. We do not know the energy distribution or the photospheric spectrum of a single star, even the sun. We do not know what spectrum corresponds to a given effective temperature, gravity, or abundances. The uncertainties in solar abundances are greater than $10 \%$, except for hydrogen, and solar abundances are the best known. It is crucial to obtain high resolution, high signal-to-noise observations of the bright stars.

Another problem arises from using a one-dimensional model as a smooth background for some other physical process when the real star actually has large scale convective motions:

- strong convection in an atmosphere and pulsation, as in a Cepheid, are physically inconsistent. Pulsation and convection must be treated together using three-dimensional radiation-hydrodynamics to investigate whether both are possible at the same time and how the pulsating star would behave if they are.

- weak, plume convection is consistent with pulsation. When the star is expanding, the convective plumes, which carry only a small percentage of the flux, and which cover only a small percentage of the area at any given time, can add to the expansion velocity and become supersonic. Shocks can form lower in the atmosphere than expected from pulsation models that ignore convection. The shock region would be three-dimensional and complex, probably with overlapping bumps. This would produce a heating and an increase in broadening velocities that would be apparent in the spectrum.

\section{Microturbulent velocity}

In the sun the microturbulent velocity is empirically found to vary from 0.5 $\mathrm{km}-\mathrm{s}^{-1}$ at the temperature minimum up $1.8 \mathrm{~km}-\mathrm{s}^{-1}$ in the deepest layers we can see. Solar spectra and predicted fluxes are computed using this depth-dependant microturbulent velocity.

This is qualitatively the behavior we expect from convective motions. The maximum convective velocity in the solar atmosphere is about 2.0 $\mathrm{km} / \mathrm{s}$ at optical depth about 4 , and the (radial) velocity slows to zero at the "surface" because there is no mass loss. If, ad hoc, we set the "surface" equal to the temperature minimum, and if we assume that the microturbulent velocity arises from, and is in some way proportional to this convective velocity, then models should incorporate depth-dependent microturbulent velocity. Because of the change in line width, all convective one-dimensional model atmospheres would then have less opacity near the surface and more at depth than is now assumed. 
Presuming that all strongly convective models have similar behavior, I propose simply scaling the solar $V$ turb- $\tau_{\text {Ross }}$ relation by the maximum convective velocity found in the atmosphere. I have tabulated this quantity for my grids of mixing-length convective models (Kurucz 1993b). It varies

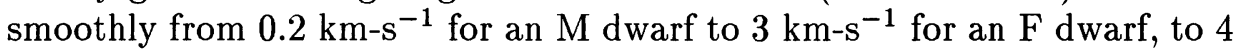
$\mathrm{km}-\mathrm{s}^{-1}$ for an $\mathrm{M}$ supergiant, and to $>10 \mathrm{~km}-\mathrm{s}^{-1}$ for an $\mathrm{F}$ supergiant. I will compute a grid where Vturb varies with Teff, $\log g$, and $\tau_{\text {Ross }}$ according to this prescription to see what happens to the models and colors. This will result in a grid in which giants automatically have high microturbulent velocities and main sequence stars have low microturbulent velocities. And in which purely radiative model atmospheres have no microturbulent velocity. Stars with plume convection would be radiative over almost all the surface so they would have bimodal line profiles with a thermal doppler core and a small blueshifted tail from the plumes. I have not yet had a chance to construct such a grid to see how it quantitatively and qualitatively differs from my current grid with constant Vturb. I should get to it later this year.

In the sun the microturbulent velocity increases in the chromosphere above the temperature minimum because of waves. The model atmosphere grids available now are model photospheres so they ignore both the heating and the increased Vturb.

Since pulsation tracks vary in Teff and gravity, models that represent pulsating stars must have Vturb and line opacities that vary with phase. In interpreting the pulsation, grids of models must be used that take into account this variation.

\section{Models and spectra for individual stars}

ATLAS12 is an opacity sampling program that I originally wrote in 1992 to allow computation of models with individual abundances. It is described in Kurucz (1993b). The reader should consult that paper for details about ATLAS12 and about my existing grids of models computed with scaled solar-abundance distribution-function line opacities. That paper has figures showing sample calculations. Castelli and Kurucz (1994) present an ATLAS12 model for Vega.

When actually working with ATLAS12 sampled fluxes, Castelli and I found that, while quite accurate for predicting the total flux, the fluxes are not accurate in intermediate or narrow bandpass intervals because the sample size is too small. I wrote a special stripped version of the spectrum synthesis program SYNTHE to generate the surface flux for the converged model using the line data from CD-ROMs 1 and 15 (Kurucz 1993a;d). Plots showing sample calculations are given in Kurucz (1995a), the complete spectrum of Arcturus with contributions by individual molecules in Kurucz 
(1994), and the complete spectrum for Sirius in Kurucz (1996b). As most of the line positions are predicted, the computed flux spectrum is not realistic when compared to a high resolution observed spectrum. It is possible to select only the subset of lines with accurate wavelengths, but many lines will be missing. This difficulty can be removed only by improving laboratory spectrum analyses. When more energy levels are known, more line positions can be computed accurately.

The spectrum is computed in pieces, typically at resolving power 500000 . It is actually computed as 17 intensity spectra spread across the disk of the star from center to limb. These pieces are merged into 17 continuous intensity spectra. Then rotationally broadened flux spectra are computed for a number of values of $v \sin i$, still at a resolving power of 500000 , by interpolating and integrating over the disk. Alternatively, the pieces can be rotationally broadened and then merged. In these calculations all information about individual lines is thrown away. To get the complete information, the calculation must be made with the full SYNTHE program. The intensity spectra from different models can be combined to mimic surface features. Of course it is not necessary to compute the whole spectrum, but only the part of interest. Any model can be used; the spectrum calculation is not coupled to ATLAS12. These high resolution spectra can be compared directly to high resolution observed spectra or they can be instrumentally broadened to compare to low resolution spectra or photometry. It is now feasible to compute grids of color indices by computing sections of spectra for every model in a grid.

ATLAS12 can be used to produce improved models for Am and Ap stars. It should be very useful for investigating diffusion effects in atmospheres. It can be used to model exciting stars for H II regions with abundances consistent with those of the H II region. These programs and line files will be distributed on CD-ROMs.

\section{Line data}

The last CD-ROM I published is Kurucz CD-ROM 23 Atomic Line Data (Kurucz and Bell 1995), which is in collaboration with Barbara Bell. It has all the laboratory and computed line data with good wavelengths sorted into one file, and then also divided into $10 \mathrm{~nm}$ or $100 \mathrm{~nm}$ blocks for convenience. It also has hyperfine splittings for the iron group.

I have made no significant progress on the line data in the last two years because of problems with office space and computers. I now have an Alpha and several Vaxes with $60 \mathrm{~GB}$ of disk, a CD writer, and a web server. There are directories for every atom and diatomic molecule. I will soon begin large scale production of atomic and molecular line data. I will continue to 
distribute the results on CD-ROMs, and I will make them available on the World Wide Web.

\section{References}

Ayres, T.R. and Testerman, L. 1981, ApJ 245, 1124-1140.

Bikmaev, I. 1994, personal communication.

Blackwell, D.E., Lynas-Gray, A.E., and Smith, G. 1995, A\&A, 296, 217.

Castelli, F. \& Kurucz, R. L. (1994) A\&A 281, 817-832.

Chan, K.L., Nordlund, $\AA$, Steffen, M., Stein, R.F. (1991) Solar Interior and Atmosphere, A.N. Cox, W.C. Livingston, and M. Matthews, eds. (Tucson: U. of Arizona Press) 223-274.

Kurucz, R. L. (1993a) Atomic data for opacity calculations. Kurucz CD-ROM No. 1.

Kurucz, R. L. (1993b) in Peculiar versus Normal Phenomena in A-type and Related Stars, M. M. Dworetsky, F. Castelli, and R. Faraggiana, eds., A.S.P. Conference Series, 8797.

Kurucz, R. L. (1993c) ATLAS9 Stellar Atmosphere Programs and 2 km/s grid. Kurucz CD-ROM No. 13.

Kurucz, R. L. (1993d) Diatomic Molecular Data for Opacity Calculations. Kurucz CDROM No. 15.

Kurucz, R. L. (1994) in Molecules in the Stellar Environment, U. G. Jørgensen, ed., Springer-Verlag, Berlin, 282-295.

Kurucz, R. L. (1995a) in Highlights of Astronomy, Vol. 10, I. Appenzeller, ed., 407-409.

Kurucz, R. L. (1995b) Ap.J.,452, 102-108.

Kurucz, R. L. (1996a) in ASP Conf. Series Vol. 108, Model Atmospheres and Stellar Spectra (ed. S. Adelman, F. Kupka, and W.W. Weiss) pp. 2-18.

Kurucz, R. L. (1996b) in ASP Conf. Series Vol. 108, Model Atmospheres and Stellar Spectra (ed. S. Adelman, F. Kupka, and W.W. Weiss) pp. 160-164.

Kurucz, R.L. and Bell, B. (1984) Atomic Line Data Kurucz CD-ROM No. 23.

Kurucz, R.L, Furenlid, I., Brault, J., and Testerman, L. (1984) Solar Flux Atlas from 296 to $1300 \mathrm{~nm}$, (Sunspot, N.M.: National Solar Observatory)

Nordlund, $\AA$. and Dravins, D. (1990) A\&A 228, 155.

Short, C.I. and Lester, J.B. (1994) ApJ 436, L365.

Topka, K.P. and Title, A.M. (1991) in Solar Interior and Atmosphere, A.N. Cox, W.C. Livingston, and M. Matthews, eds. (Tucson: U. of Arizona Press) 727-747.

\section{DISCUSSION}

CLAUDE MEGESSIER: You mentioned the effects that affect the Balmer line wings and lead to errors in stellar effective temperature determinations through the Balmer line fitting. The advantage of the Infrared Flux Method is to take into account the integrated flux over the whole wavelength range and it does not consider some features in the spectra. Then the near-infrared region used by the method plays an important part and it is essential to know the physical phenomena that could affect it.

ROBERT KURUCZ: The Infrared Flux Method may be the only 'safe' method, although there is a systematic error. Because of flux conservation, an increased UV flux would be balanced 
by a decreased IR flux. This would probably be a few degrees error in effective temperature.

JOHANNES ANDERSEN: From your talk and the previous one, I gather that the useful days of 1-D atmosphere models are numbered. Do you think their shortcomings are the reasons for the problems with synthetic colours ('Hyades anomaly,' etc.) and abundances from temperature-sensitive lines ( $\mathrm{Li}$ I, OI triplet)? And, as a comment, some years ago Dravins and Nordlund derived the kind of different convection patterns in $F$ and later-type stars that you mentioned from an analysis of line bisectors in such stars.

ROBERT KURUCZ: There should be an inconsistency between ultraviolet and red or IR colors. And yes, there should be effects in abundance analysis.

UNKNOWN: Although we cannot do 3-D with good resolution yet (but soon), 2-D hydrodynamic simulations of stellar convection give higher velocities than mixing length theory. This is because mixing length is local, and plumes are accelerated over their path. Thus, microturbulence velocities inferred from mixing length should be used with some skepticism.

DIFFERENT UNKNOWN: A depth-dependent microturbulence would go part of the way in reducing the high $\mathrm{FeI}$ abundance obtained by the Oxford group.

ROBERT KURUCZ: Yes, I think they did a test with depth-dependent microturbulent velocity. 